\title{
ASYMPTOTICS OF SOLUTIONS TO A CLASS OF LINEAR DIFFERENTIAL EQUATIONS
}

\author{
N.N. KONECHNAYA, K.A. MIRZOEV
}

\begin{abstract}
In the paper we find the leading term of the asymptotics at infinity for some fundamental system of solutions to a class of linear differential equations of arbitrary order $\tau y=\lambda y$, where $\lambda$ is a fixed complex number. At that we consider a special class of ShinZettl type and $\tau y$ is a quasi-differential expression generated by the matrix in this class. The conditions we assume for the primitives of the coefficients of the quasi-differential expression $\tau y$, that is, for the entries of the corresponding matrix, are not related with their smoothness but just ensures a certain power growth of these primitives at infinity. Thus, the coefficients of the expression $\tau y$ can also oscillate. In particular, this includes a wide class of differential equations of arbitrary even or odd order with distribution coefficients of finite order. Employing the known definition of two quasi-differential expressions with nonsmooth coefficients, in the work we propose a method for obtaining asymptotic formulae for the fundamental system of solutions to the considered equation in the case when the left hand side of this equations is represented as a product of two quasi-differential expressions.

The obtained results are applied for the spectral analysis of the corresponding singular differential operators. In particular, assuming that the quasi-differential expression $\tau y$ is symmetric, by the known scheme we define the minimal closed symmetric operator generated by this expression in the space of Lebesgue square-integrable on $[1,+\infty)$ functions (in the Hilbert space $\mathcal{L}^{2}[1,+\infty)$ ) and we calculate the deficiency indices for this operator.
\end{abstract}

Keywords: Quasi-derivative, quasi-differential expression, the main term of asymptotic of the fundamental system of solutions, minimal closed symmetric differential operator, deficiency numbers.

Mathematics Subject Classification: 34E05, 34L05

\section{INTRODUCTION}

Let the entries of a matrix $F=\left(f_{j k}\right)$, the complex-valued functions $f_{j k}, j, k=1,2 \ldots, m$, $m>1$, be well-defined and measurable on an interval $(a, b),-\infty \leqslant a<b \leqslant+\infty$ and satisfy the following conditions:

1) $f_{j k}=0$ almost everywhere in $(a, b)$ as $2 \leqslant j+1<k \leqslant m$ and $f_{j, j+1} \neq 0$ almost everywhere on $(a, b)$ as $1 \leqslant j \leqslant m-1$;

2 ) the functions $f_{j k}$ are locally Lebesgue integrable in $(a, b)$ for all $1 \leqslant j, k \leqslant m$, that is, $f_{j k}$ are locally Lebesgue integrable in each segment $[\alpha, \beta] \subset(a, b)\left(f_{j k} \in \mathcal{L}_{l o c}^{1}(a, b)\right)$.

N.N. Konechnaya, K.A. Mirzoev, Asymptotics of solutions to a Class of linear differential EQUATIONS.

(C) Konechanaya N.N., Mirzoev K.A. 2017.

The first author was supported by a grant of Russian Science (project no. 17-11-01215).

Submitted May 25, 201\%. 
We define the quasi-derivatives $y^{[j]}(0 \leqslant j \leqslant m-1)$ of a given function $y$ by means of the matrix $F$ by letting $y^{[0]}:=y$ and

$$
y^{[j]}:=\left(f_{j, j+1}\right)^{-1}\left[\left(y^{[j-1]}\right)^{\prime}-\sum_{k=1}^{j} f_{j k} y^{[k-1]}\right], \quad j=1,2, \ldots, m-1,
$$

assuming that $y^{[k-1]}(k=1,2, \ldots, j)$ are already defined and are absolutely continuous functions on each compact set $[\alpha, \beta] \subset(a, b)\left(y^{[k-1]} \in A C_{l o c}(a, b)\right)$. We defined also a quasi-differential expression $\tau y$ by means of the matrix $F$ letting

$$
\tau y:=i^{m}\left[\left(y^{[m-1]}\right)^{\prime}-\sum_{k=1}^{m} f_{m k} y^{[k-1]}\right] .
$$

The natural domain $\mathcal{D}(\tau)$ of the expression $\tau$ is the set of all complex-valued functions $y$, for which there exist locally absolutely continuous quasi-derivatives $y^{[j]}$ up $(m-1)$ th order and it is obvious that $\tau y \in \mathcal{L}_{\text {loc }}^{1}(a, b)$ for each $y \in \mathcal{D}(\tau)$.

We consider the differential equation

$$
\tau y=\lambda y,
$$

where $\lambda \in \mathbb{C}$ is a parameter. This equation is equivalent to the system of the first order linear differential equations

$$
\mathbf{y}^{\prime}=(F+\Lambda) \mathbf{y}
$$

where $\mathbf{y}$ is the vector column $\mathbf{y}:=\operatorname{colon}\left(y^{[0]}, y^{[1]}, \ldots, y^{[m-1]}\right)$, and the entries of a square matrix $\Lambda=\left(\lambda_{i j}\right)$ of the size $m \times m$ are defined by the identities $\lambda_{m 1}:=i^{-m} \lambda$ and $\lambda_{i j}:=0$ for other values $i$ and $j$. Scalar equation (3) and system (4) are equivalent in the sense that if $y$ is a solution to equation (3), then $\mathbf{y}=\operatorname{colon}\left(y^{[0]}, y^{[1]}, \ldots, y^{[m-1]}\right)\left(y=y^{[0]}\right)$ solves system (4) and vice versa, if $\mathbf{y}=\operatorname{colon}\left(y_{0}, y_{1}, \ldots, y_{m-1}\right)$ is a solution to system (4), then $y=y_{0}$ solves equation (3) and $y_{k}=y^{[k]}(k=0,1, \ldots m-1)$.

We note that Condition 1) allows us to define the quasi-derivatives of a function $y \in \mathcal{D}(\tau)$ and the scalar linear quasi-differential expression $\tau y$ (of order $m$ ) in terms of the matrix $F$ by formulae (1) and (2), while Condition 2) ensures the validity of the theorem on the unique solvability of the Cauchy problem for system of equations (4) posed in an arbitrary point of the segment $(a, b)$. Thus, under Conditions 1) and 2), the theorem on the unique solvability of the Cauchy problem is valid for equation (3).

In a special literature, the matrices satisfying Conditions 1) and 2) are called matrices of ShinZettl type and the class of these matrices is denoted by the symbol $Z_{m}(a, b)$ (see, for instance, [1, Sect. I] or [2]) or by the symbol $\mathcal{S}_{m}(a, b)$ (see [3]). The definition of the quasi-derivatives and of the quasi-differential expression are borrowed from these works.

In what follows we assume that $a=1$ and $b=+\infty$. In Section 2 we define a subclass of the matrices in $Z_{m}[1,+\infty)$ and generated by them quasi-differential equations (3) studied in the present work. In Section 3 we prove the theorem on an asymptotics at infinity for some fundamental system of solutions to this class of equations. Following work [2] (see also [4]), in Section 4 we define the product of two quasi-differential expression and we propose a method allowing us to obtain asymptotic formulae for the solutions to equation (3) in the case, when the left hand side of this equations is represented as the product of two expressions in the class defined in Section 2. Posing additional assumptions for the matrix $F$ ensuring the symmetricity (formal self-adjointness) of the expression $\tau y$, see [1, Sect. I], in Section 5 we define the minimal closed symmetric operator generated by this expression in the space of Lebesgue square integrable functions in $\left[1,+\infty\right.$ ) (in the Hilbert space $\mathcal{L}^{2}[1,+\infty)$ ). The results obtained in Section 3 is applied for determining the deficiency index of this operator. 
The results of this paper were partially announced in work [5] without the proofs.

\section{Construction of a special matrix $F$}

2.1. Let $m=2 n$. We introduce the matrix $F_{2 n}(=: F)$ as

$$
F_{2 n}=\left(\begin{array}{ccccccccccc}
0 & 1 & 0 & . & . & 0 & 0 & 0 & . & . & 0 \\
0 & 0 & 1 & . & . & 0 & 0 & 0 & . & . & 0 \\
. & . & . & . & . & . & . & . & . & . & . \\
0 & 0 & 0 & . & . & 1 & 0 & 0 & . & . & 0 \\
f_{n, 1} & f_{n, 2} & f_{n, 3} & . & . & f_{n, n} & f_{n, n+1} & 0 & . & . & 0 \\
f_{n+1,1} & f_{n+1,2} & f_{n+1,3} & . & . & f_{n+1, n} & f_{n+1, n+1} & 1 & . & . & 0 \\
\cdot & \cdot & . & . & . & . & . & . & . & . & . \\
f_{2 n-1,1} & f_{2 n-1,2} & f_{2 n-1,3} & . & . & f_{2 n-1, n} & f_{2 n-1, n+1} & 0 & . & . & 1 \\
f_{2 n, 1} & f_{2 n, 2} & f_{2 n, 3} & . & . & f_{2 n, n} & f_{2 n, n+1} & 0 & . & . & 0
\end{array}\right) .
$$

We assume that the entries $f_{j k}$ of the matrix $F_{2 n}$ satisfy Conditions 1) and 2) of Section 1 , that is,

(A) $f_{j k} \in \mathcal{L}_{\text {loc }}^{1}[1,+\infty)$ as $n \leqslant j \leqslant 2 n, 1 \leqslant k \leqslant n+1$, and $f_{n, n+1} \neq 0$ almost everywhere in $[1,+\infty)$.

In addition, we assume that

$(B)$ there exists a number $\nu \geqslant 0$, complex numbers $\alpha_{j k}$ and complex-valued functions $\beta_{j k}(x)$ such that $\alpha_{n, n+1} \neq 0$ and for all $x \geqslant 1$ the entries $f_{j k}$ are of the form

$$
\begin{array}{ll}
f_{n, n+1}(x):=x^{-2 n-\nu}\left(\alpha_{n, n+1}+\beta_{n, n+1}(x)\right) ; & \\
f_{n j}(x):=x^{-n+j-1}\left(\alpha_{n j}+\beta_{n j}(x)\right), & j=1, \ldots n ; \\
f_{n+k, n+1}(x):=x^{-k}\left(\alpha_{n+k, n+1}+\beta_{n+k, n+1}(x)\right), & k=1, \ldots n ; \\
f_{n+k, j}(x):=x^{n+\nu-k+j-1}\left(\alpha_{n+k, j}+\beta_{n+k, j}(x)\right), & k=1, \ldots n, j=1, \ldots n .
\end{array}
$$

We denote by $D:=\operatorname{diag}\left(d_{1}, d_{2}, \ldots, d_{2 n}\right)$ the diagonal matrix function with the entries

$$
d_{k}(x):=x^{-k+\frac{1}{2}}, \quad d_{k+n}(x):=x^{n+\nu-k+\frac{1}{2}}, \quad k=1, \ldots, n .
$$

Making the change $\mathbf{y}=D \mathbf{Y}$ in system (4) (with $F=F_{2 n}$ ), it is easy to see that the new unknown function $\mathbf{Y}$ satisfies the system of equations

$$
\mathbf{Y}^{\prime}=\left(D^{-1} F_{2 n} D+D^{-1} \Lambda D-D^{-1} D^{\prime}\right) \mathbf{Y}
$$

where for the entries $\tilde{f}_{i j}$ of the matrix $D^{-1} F_{2 n} D$ the formulae

$$
\tilde{f}_{i j}=d_{i}^{-1} f_{i j} d_{j}, \quad i, j=1,2, \ldots 2 n,
$$

hold true and all the entries of the matrix $D^{-1} \Lambda D$ vanish except for the entry in the left lower corner, which is equal to $x^{-1}(-1)^{n} \lambda / x^{\nu}$. Moreover,

$$
D^{-1} D^{\prime}=x^{-1} \operatorname{diag}\left(-\frac{1}{2},-\frac{3}{2}, \ldots,-n+\frac{1}{2}, n+\nu-\frac{1}{2}, n+\nu-\frac{3}{2}, \ldots, \nu+\frac{1}{2}\right) .
$$

This arguing imply easily that the unknown vector function $\mathbf{Y}$ satisfies the system of equations

$$
x \frac{d \mathbf{Y}}{d x}=(A+B(x)) \mathbf{Y},
$$

where $A=A_{1}+A_{2}+A_{3}$ is a constant matrix and the matrix $A_{1}$ is of the same form as $F_{2 n}$ with the only difference that $f_{j k}$ are replaced by $\alpha_{j k}$ and $A_{2}$ is the diagonal matrix defined by 
the identity

$$
A_{2}=\operatorname{diag}\left(\frac{1}{2}, \frac{3}{2}, \ldots, n-\frac{1}{2}, \frac{1}{2}-n-\nu, \frac{3}{2}-n-\nu, \ldots,-\frac{1}{2}-\nu\right)
$$

and $A_{3}$ is the matrix, which is equal to $\Lambda$ (see (40) as $\nu=0$ and to the zero matrix as $\nu>0$. Moreover, in (5), the non-zero entries $b_{j k}(x)$ of the matrix function $B(x)$ are such that $b_{j k}(x)=\beta_{j k}(x)$ as $n \leqslant j \leqslant 2 n, 1 \leqslant k \leqslant n+1$, except for the entry $b_{2 n, 1}(x)$, and

$$
b_{2 n, 1}(x)= \begin{cases}\beta_{2 n, 1}(x), & \text { if } \nu=0 \\ \beta_{2 n, 1}(x)+\frac{(-1)^{n} \lambda}{x^{\nu}}, & \text { if } \nu>0 .\end{cases}
$$

2.2. Let $m=2 n+1$. We define the matrix $F_{2 n+1}(=: F)$ as

$$
F_{2 n+1}=\left(\begin{array}{cccccccccccc}
0 & 1 & 0 & . & . & 0 & 0 & 0 & 0 & . & . & 0 \\
0 & 0 & 1 & . & . & 0 & 0 & 0 & 0 & . & . & 0 \\
\cdot & . & . & . & . & . & . & . & . & . & . & . \\
0 & 0 & 0 & . & . & 1 & 0 & 0 & 0 & . & . & 0 \\
0 & 0 & 0 & . & . & 0 & f_{n, n+1} & 0 & 0 & . & . & 0 \\
f_{n+1,1} & f_{n+1,2} & f_{n+1,3} & . & . & f_{n+1, n} & f_{n+1, n+1} & f_{n+1, n+2} & 0 & . & . & 0 \\
f_{n+2,1} & f_{n+2,2} & f_{n+2,3} & . & . & f_{n+2, n} & f_{n+2, n+1} & 0 & 1 & . & . & 0 \\
\cdot & . & . & . & . & . & . & . & . & . & . & \\
f_{2 n, 1} & f_{2 n, 2} & f_{2 n, 3} & . & . & f_{2 n, n} & f_{2 n, n+1} & 0 & 0 & . & . & 1 \\
f_{2 n+1,1} & f_{2 n+1,2} & f_{2 n+1,3} & . & . & f_{2 n+1, n} & f_{2 n+1, n+1} & 0 & 0 & . & . & 0
\end{array}\right)
$$

In the considered case we assume that all entries $f_{j k}$ of the matrix $F_{2 n+1}$ satisfy the conditions (A) $f_{n, n+1}, f_{n+1, n+2}, f_{j k} \in \mathcal{L}_{l o c}^{1}[1,+\infty)$ as $n+1 \leqslant j \leqslant 2 n+1,1 \leqslant k \leqslant n+1$, and $f_{n, n+1} \neq 0$, $f_{n+1, n+2} \neq 0$ almost everywhere in $[1,+\infty)$,

and

$(B)$ there exists a number $\nu \geqslant 0$, complex numbers $\alpha_{j k}$ and complex-valued functions $\beta_{j k}(x)$ such that $\alpha_{j, j+1} \neq 0$ as $j=n, n+1$ and for all $x \geqslant 1$ the entries $f_{j k}$ are of the form

$$
\begin{array}{ll}
f_{n, n+1}(x):=x^{-n-\frac{\nu}{2}-\frac{1}{2}} \quad\left(\alpha_{n, n+1}+\beta_{n, n+1}(x)\right) ; & \\
f_{n+1, n+2}(x):=x^{-n-\frac{\nu}{2}-\frac{1}{2}} \quad\left(\alpha_{n+1, n+2}+\beta_{n+1, n+2}(x)\right) ; & \\
f_{n+1, n+1}(x):=x^{-1}\left(\alpha_{n+1, n+1}+\beta_{n+1, n+1}(x)\right) ; & j=1, \ldots, n ; \\
f_{n+1, j}(x):=x^{\frac{\nu}{2}+j-\frac{3}{2}}\left(\alpha_{n+1, j}+\beta_{n+1, j}(x)\right), & \\
f_{n+1+j, n+1}(x):=x^{\frac{\nu}{2}+n-j-\frac{1}{2}}\left(\alpha_{n+1+j, n+1}+\beta_{n+1+j, n+1}(x)\right), & j=1, \ldots, n ; \\
f_{n+1+j, k}(x):=x^{\nu+n-j+k-1}\left(\alpha_{n+1+j, k}+\beta_{n+1+j, k}(x)\right), & j=1, \ldots, \quad k=1, \ldots, n .
\end{array}
$$

Moreover, now the entries $d_{k}$ of the matrix $D:=\operatorname{diag}\left(d_{1}, d_{2}, \ldots, d_{2 n+1}\right)$ in the change $\mathbf{y}=D \mathbf{Y}$ are defined by the identities

$$
d_{k}(x):=x^{-k+\frac{1}{2}}, \quad d_{n+1}(x):=x^{\frac{\nu}{2}}, \quad d_{n+1+k}(x):=x^{\nu+n-k+\frac{1}{2}}, \quad k=1,2, \ldots, n .
$$

By means of such change system (4) (with $F=F_{2 n+1}$ ) is again transformed to the system of the form

$$
\mathbf{Y}^{\prime}=\left(D^{-1} F_{2 n+1} D+D^{-1} \Lambda D-D^{-1} D^{\prime}\right) \mathbf{Y}
$$

for the unknown vector function $\mathbf{Y}$. We note that in this case the entries $\tilde{f}_{i j}$ of the matrix $D^{-1} F_{2 n+1} D$ can be found, as before, by the formulae

$$
\tilde{f}_{i j}=d_{i}^{-1} f_{i j} d_{j}, \quad i, j=1,2, \ldots 2 n+1,
$$


and all entries of the matrix $D^{-1} \Lambda D$ are equal to zero except the entry in the left lower corner, which is equal to $x^{-1} i(-1)^{n+1} \lambda / x^{\nu}$. Moreover,

$$
D^{-1} D^{\prime}=x^{-1} \operatorname{diag}\left(-\frac{1}{2},-\frac{3}{2}, \ldots,-n+\frac{1}{2}, \frac{\nu}{2}, n+\nu-\frac{1}{2}, n+\nu-\frac{3}{2}, \ldots, \nu+\frac{1}{2}\right) .
$$

Thus, in this case the unknown vector function $\mathbf{Y}$ also satisfies system of equations (5), where $A=A_{1}+A_{2}+A_{3}$ and the matrices $A_{1}$ and $A_{3}$ are formed in the same way as in the case $m=2 n$ with $F_{2 n}$ replaced by $F_{2 n+1}$, and the matrix $A_{2}$ is of the form

$$
A_{2}=\operatorname{diag}\left(\frac{1}{2}, \frac{3}{2}, \ldots, n-\frac{1}{2},-\frac{\nu}{2}, \frac{1}{2}-n-\nu, \frac{3}{2}-n-\nu, \ldots,-\frac{1}{2}-\nu\right) .
$$

Moreover, in (5), the non-zero entries $b_{j k}(x)$ in $B(x)$ are such that $b_{j, j+1}(x)=\beta_{j, j+1}(x)$ as $j=n, n+1, b_{j k}(x)=\beta_{j k}(x)$ as $n+1 \leqslant j \leqslant 2 n+1,1 \leqslant k \leqslant n+1$, except the entry $b_{2 n+1,1}(x)$ and

$$
b_{2 n+1,1}(x)= \begin{cases}\beta_{2 n+1,1}(x), & \text { if } \nu=0 \\ \beta_{2 n+1,1}(x)+i \frac{(-1)^{n+1} \lambda}{x^{\nu}}, & \text { if } \nu>0 .\end{cases}
$$

Condition (C) we formulate below is the same for both cases $m=2 n$ and $m=2 n+1$. Let $r+1$ be the maximal multiplicity of the characteristic roots of the matrix $A$ and let

$(C)$ the matrices $A$ and $B(x)$ are such that

$$
\int_{1}^{\infty} \frac{(\ln x)^{r}}{x}\|B(x)\| d x<\infty
$$

where $\|B(x)\|$ stands for the sum of the absolute values of all entries in the matrix $B(x)$.

Remark 1. The matrices $F_{2 n}$ and $F_{2 n+1}$ are chosen so that each differential expression of the form

$$
\tau y=\sum_{k=0}^{[m / 2]}\left(p_{k} y^{(k)}\right)^{(k)}+i \sum_{k=0}^{[(m-1) / 2]}\left[\left(q_{k} y^{(k+1)}\right)^{(k)}+\left(q_{k} y^{(k)}\right)^{(k+1)}\right],
$$

where $[a]$ is the maximal integer number not exceeding $a$, and $m=2 n$ or $m=2 n+1$, with sufficiently smooth coefficients is generated by the matrices of such form and all the entries of these matrices vanish except for the necessary non-zero entries (cf. the form of matrices $F_{2 n}, F_{2 n+1}$ and Condition $\left.(A)\right)$ and possibly the entries at the secondary diagonal and at two diagonals to the right and to the left of the secondary one and the non-zero entries are smooth functions, for details see [1, Append. A]. Moreover, the Shin-Zettl matrices $F_{2 n}$ and $F_{2 n+1}$ constructed in [1] and generating expression (7)) are such that if we replace the smoothness of the entries in these matrices by their local integrability and if the derivatives in formulae (1) and (2) are treated in the distribution sense, then we can open the brackets and a regular generalized function $\tau y$ in (2) for $y \in \mathcal{D}(\tau)$ is represented as (7) in terms of the theory of generalized functions. At that we stress that the coefficients $p_{k}$ and $q_{k}$ in expression (7) should be only locally integrable (see [6], [7]).

It was announced in work [3] that a wide class of expressions of form (7) of arbitrary order with distribution coefficients is also covered by the class of quasi-differential expression generated by the Shin-Zettl matrices of form $F_{2 n}$ or $F_{2 n+1}$.

Remark 2. The definition of quasi-derivatives and quasi-differential expression via formulae (1) and (2) by means of the matrices $F_{2 n}$ and $F_{2 n+1}$ allows us to state that $y^{[j]}=y^{(j)}$ as $0 \leqslant j \leqslant n-1$, that is, the entries of these matrices not equalling to one are involved only in the definition of $y^{[j]}$ as $n \leqslant j \leqslant m-1$ and $\tau y$. Using this fact, we can show that in the case when 
the matrices $F_{2 n}$ and $F_{2 n+1}$ generate differential expression (7), as $y \in \mathcal{D}(\tau)$, the expressions $p_{k} y^{(k)}(0 \leqslant k \leqslant[m / 2])$ and $q_{k} y^{(k+1)}(0 \leqslant k \leqslant[(m-1) / 2])$ are regular generalized functions. Thus, as $y \in \mathcal{D}(\tau)$, the terms in expression (7) are generalized derivatives of regular generalized functions and their sum $\tau y$ is a regular generalized function.

\section{LEADING TERM in THE ASYMPtotics OF SOlUTIONS}

3.1. In what follows we make use of the following statement proved in [8], see also [9]; [10, Ch. III, Prob. 35]; [11, Ch. IV].

Lemma 1. We consider the system of differential equations

$$
V^{\prime}(t)=(A+R(t)) V(t)
$$

where the matrix $A$ is constant and the canonical form of the matrix $A$ has Jordan blocks $J_{k}$, $k \geqslant 1$, and maximal number of the rows for all blocks $J_{k}$ is equal to $r+1,(r \geqslant 1)$. Assume that

$$
\int_{1}^{\infty} t^{r}\|R(t)\| d t<\infty .
$$

Let $z$ be the characteristic root $A$ and let the equation

$$
V^{\prime}(t)=A V(t)
$$

has a solution of the form

$$
e^{z t} t^{k} C+O\left(e^{z t} t^{k-1}\right)
$$

where $C$ is a constant vector. Then equation (8) has a solution $\varphi$ such that

$$
\varphi(t)=e^{z t} t^{k}(C+o(1)), \quad t \rightarrow+\infty .
$$

3.2. We prove the following theorem.

Theorem 1. Let the entries of the matrix $F$ satisfy conditions $(A)-(C)$ and $z_{1}, z_{2}, \ldots, z_{q}$, $z_{q+1}, \ldots, z_{q+j}$ be different characteristic roots of the matrix $A$ and $z_{1}, z_{2}, \ldots, z_{q}$ are simple roots; as $1 \leqslant p \leqslant j$, the multiplicity of the root $z_{q+p}$ is equal to $r_{p}$. Then equation (3) has a fundamental system of solutions $y_{k}(x)$ such that as $x \rightarrow+\infty$

$$
y_{k}(x)=c_{k} x^{z_{k}-\frac{1}{2}}(1+o(1)), \quad k=1,2, \ldots, q,
$$

and as $k=q, q+r_{1}, \ldots, q+r_{1}+\ldots+r_{j-1}$,

$$
y_{k+i}(x)=c_{k+i} x^{z_{q+p}-\frac{1}{2}}(\ln x)^{i-1}(1+o(1)), \quad i=1, \ldots, r_{p}, \quad \text { if } k=q+r_{1}+\ldots+r_{p-1},
$$

where $c_{1}, c_{2}, \ldots, c_{m}$ are arbitrary non-zero constants.

Proof. The structure of the matrix $A$ both as $m=2 n$ and $m=2 n+1$ is such that $a_{j, j+1} \neq 0$ as $1 \leqslant j \leqslant m-1$ and $a_{j k}=0$ as $2 \leqslant j+1<k \leqslant m$, see Section 2 . This is an eigenvector associated with an eigenvalue is uniquely determined by its first coordinate. Thus, the geometric multiplicity of each eigenvalue of the matrix $A$ is equal to one. In other words, with each eigenvalue of the matrix $A$, only one Jordan block in its canonical form is associated. This is why the maximal dimension of a Jordan block in the canonical form of the matrix $A$, the number $r+1$ in Lemma 1 coincides with the maximal multiplicity of the eigenvalues of the matrix $A$, the number $r+1$ in Condition $(\mathrm{C})$.

We transform system of differential equations (5) by letting $x=e^{t}$. Then this system casts into form (8), where $V(t)=\mathbf{Y}\left(e^{t}\right), R(t)=B\left(e^{t}\right)$. Moreover, if the matrices $A$ and $B(x)$ satisfy Condition (C), then the matrix $R(t)$ satisfies condition (9) of Lemma 1. If $z_{1}, z_{2}, \ldots, z_{q}$ are different characteristic roots of the matrix $A$, then system of equations with constant coefficients 
(10) has the solutions $e^{z_{k} t} C_{k}$, where $C_{k}$ is an eigenvector corresponding to the eigenvalue $z_{k}$, $(1 \leqslant k \leqslant q)$. Applying Lemma 1, we find that system (8) has solutions of the form

$$
e^{z_{k} t}\left(C_{k}+o(1)\right), \quad t \rightarrow+\infty
$$

Thus, making the inverse change $t=\ln x$ and taking into consideration the transformation $\mathbf{y}=D \mathbf{Y}$, we find that system (4) has solutions represented as

$$
x^{z_{k}} D\left(C_{k}+o(1)\right), \quad x \rightarrow+\infty .
$$

Therefore, the first coordinate of this solution, the solution $y_{k}$ of equation (3), is represented as (11), where $c_{k}$, the first coordinate of the eigenvector $C_{k}$, is non-zero.

Let $z_{q+p}$ be the characteristic root of matrix $A$ of multiplicity $r_{p}$. As we know, only one Jordan block in the canonical expansion of the matrix $A$ is associated with this characteristic root and this is why the dimension of this block is equal to $r_{p}$ and it does not exceed the number $r+1$. This implies that system of equations 10 has solution represented as

$$
e^{z_{q+p} t} C_{q+p} \quad \text { and } \quad e^{z_{q+p} t} t^{k} C_{q+p}+O\left(e^{z_{q+p} t} t^{k-1}\right), \quad k=1,2, \ldots, r_{p}-1,
$$

where $C_{q+p}$ is an eigenvector associated with the eigenvalue $z_{q+p}$. Applying Lemma 1 one more time and arguing as in the case of a simple eigenvalue, we can easily find that equation (3) has exactly $r_{p}$ solutions represented as $(12)$ and associated with the eigenvalue $z_{q+p}$. It remains to consider the set of solutions to equation (3) represented as (11) and (12) and associated with all eigenvalues of the matrix $A$. It is obvious that this set forms a fundamental system of solutions to this equation.

We denote by $\mathfrak{F}(z, \nu)$ the characteristic polynomial of the matrix $A$. Theorem 1 implies the following statement.

Corollary 1. Assume that the entries of the number of linearly independent solutions to equations (3) belonging to the space $\mathcal{L}_{2}[1,+\infty)$ is equal to

1) the number of the roots of the polynomial $\mathfrak{F}(z, \nu)$ located in the domain $\operatorname{Re} z<0$ as $\nu>0$; this number is independent of $\lambda$;

2) the number of the roots of the polynomial $\mathfrak{F}(z, 0)-\lambda$ located in the domain $\operatorname{Re} z<0$ as $\nu=0$.

Remark 3. Let $y(x)$ be an arbitrary solution to equation (3), whose asymptotic behavior is determined by Theorem 1. The given proof of this theorem allows us to obtain the leading term of the asymptotics at infinity for the quasi-derivatives $y^{[j]}(x)$ of the function $y(x)$. In order to do this, the $(j+1)$ th component of the corresponding eigenvector should be non-zero. In particular, in view of Remark 2, we obtain that in some cases, the asymptotic formulae in Theorem 1 can be differentiated up to $(n-1)$ th order.

Remark 4. The matrix function $B(x)$ is not explicitly involved in the asymptotic formulae in Theorem 1, it is just sufficient this matrix to satisfy Condition (C). Assume that the entries $f_{j k}(x)$ of the matrix $F$ (equal to $F_{2 n}$ or to $F_{2 n+1}$ ) are chosen so that the corresponding differential expression $\tau y$ is represented as (7) and at that $B(x)$ is a zero matrix, see Remark 1 . In this situation, the analysis of expression $\tau y$ shows that in the case $m=2 n$, if the coefficients $p_{k}$, $k=0,1, \ldots, n$, and $q_{k}, k=0,1, \ldots, n-1$, satisfy Condition (B), then there exist complex numbers $a_{k}, k=0,1, \ldots, n$, and $b_{k}, k=0,1, \ldots, n-1$, such that the roots of the polynomial 
$\mathfrak{F}(z, \nu)$ coincide, as $\nu>0$, with the roots of the polynomial

$$
\begin{aligned}
\mathfrak{F}_{2 n}(z, \nu)= & a_{0}+\sum_{k=1}^{n} a_{k} \prod_{j=0}^{k-1}\left[\left(z+\frac{\nu}{2}\right)^{2}-\left(\frac{\nu+1}{2}+j\right)^{2}\right] \\
& +2 i\left(z+\frac{\nu}{2}\right)\left\{b_{0}+\sum_{k=1}^{n-1} b_{k} \prod_{j=0}^{k-1}\left[\left(z+\frac{\nu}{2}\right)^{2}-\left(\frac{\nu+1}{2}+j\right)^{2}\right]\right\} .
\end{aligned}
$$

The similar statement is true as $m=2 n+1$, that is, there exists the polynomial $\mathfrak{F}_{2 n+1}(z, \nu)$ of the form

$$
\mathfrak{F}_{2 n+1}(z, \nu)=\mathfrak{F}_{2 n}(z, \nu)+2 i\left(z+\frac{\nu}{2}\right) b_{n} \prod_{j=0}^{n-1}\left[\left(z+\frac{\nu}{2}\right)^{2}-\left(\frac{\nu+1}{2}+j\right)^{2}\right]
$$

such that the eigenvalues of the matrix $A$ coincide, as $\nu>0$, with the roots of this polynomial, for more details on the polynomials $\mathfrak{F}_{2 n}$ and $\mathfrak{F}_{2 n+1}$ see [6], [7].

\section{ProduCT OF QUASI-DIFFERENTIAL EXPRESSIONS}

4.1. In the present section we equip the quasi-derivatives $y^{[j]}$, the quasi-differential expression $\tau y$, the matrices $D, A, B(x)$ and the polynomials $\mathfrak{F}(z, \nu)$ (see Sections 2 and 3 ) by the subscript $F$ stressing in this way that they are constructed in terms of the matrix $F$. Following works [2] and [4], first we define the product of two quasi-differential expressions. Let $\mathcal{F} \in Z_{m}(a, b)$, $\mathcal{G} \in Z_{l}(a, b), m, l>1$, and

$$
\mathcal{H}:=\left(\begin{array}{cc}
\mathcal{G} & M \\
O_{m \times l} & \mathcal{F}
\end{array}\right)
$$

where $M$ is the matrix of size $l \times m$; all its entries are zero except that in the left lower corner, which is equal to 1 , and $O_{m \times l}$ is the zero matrix of size $m \times l$. It is obvious that $\mathcal{H} \in Z_{m+l}(a, b)$, that is, the matrix $\mathcal{H}$ satisfies Conditions 1) and 2) of Section 1. In view of the definition of the quasi-derivatives and the quasi-differential expression, see formulae(1) and (2) in Section 1, it is easy to establish that

$$
\begin{aligned}
y_{\mathcal{H}}^{[0]} & :=y, \quad y_{\mathcal{H}}^{[j]}:=y_{\mathcal{G}}^{[j]}, \quad j=1, \ldots, l-1, \\
y_{\mathcal{H}}^{[l]} & :=\left(y_{\mathcal{G}}^{[l-1]}\right)^{\prime}-\sum_{k=1}^{l} g_{l k} y_{\mathcal{G}}^{[k-1]}, \\
y_{\mathcal{H}}^{[l+r]} & :=\left(y_{\mathcal{G}}^{[l]}\right)_{\mathcal{F}}^{[r]}, \quad r=1, \ldots, m-1,
\end{aligned}
$$

and

$$
\tau_{\mathcal{H}} y:=i^{m+l}\left[\left(y_{\mathcal{H}}^{[l+m-1]}\right)^{\prime}-\sum_{k=1}^{m} f_{m k} y_{\mathcal{H}}^{[l+k-1]}\right] .
$$

By these formulae we see that the domain $\mathcal{D}\left(\tau_{\mathcal{H}}\right)$ of the expression $\tau_{\mathcal{H}}$ is given by the identity

$$
\mathcal{D}\left(\tau_{\mathcal{H}}\right)=\left\{y \mid y \in \mathcal{D}\left(\tau_{\mathcal{G}}\right) \text { and } \tau_{\mathcal{G}} y \in \mathcal{D}\left(\tau_{\mathcal{F}}\right)\right\},
$$

and moreover,

$$
\tau_{\mathcal{H}} y=\tau_{\mathcal{F}}\left(\tau_{\mathcal{G}} y\right) \quad \text { as } \quad y \in \mathcal{D}\left(\tau_{\mathcal{H}}\right)\left(=\mathcal{D}\left(\tau_{\mathcal{F}} \tau_{\mathcal{G}}\right)\right)
$$

for details see [2], 4].

In the case $\tau=\tau_{\mathcal{H}}$ equation (3) is, as usually, equivalent to system (4), that is,

$$
\mathbf{y}^{\prime}=(\mathcal{H}+\Lambda) \mathbf{y}
$$


where $\mathbf{y}$ is the unknown vector column with $m+l$ components and the entries of the square matrix $\Lambda=\left(\lambda_{i j}\right)$ of the size $(m+l) \times(m+l)$ are defined by the identities $\lambda_{m+l, 1}:=i^{-m-l} \lambda$ and $\lambda_{i j}:=0$ for all other values $i$ and $j$.

4.2. Now let the matrices $\mathcal{G} \in Z_{l}[1,+\infty)$ and $\mathcal{F} \in Z_{m}[1,+\infty)$ have the same structrure as the matrix $F$ in Section 2 and therefore, they satisfy Condition (A). Let $\nu_{1}, \nu_{2}>0$ be some constants and the entries $g_{j k}$ and $f_{j k}$ of the matrices $\mathcal{G}$ and $\mathcal{F}$ are determined by the identities similar to the identities in Condition (B) in Section 2 with the parameters $\nu_{1}$ and $\nu_{2}$, respectively.

We define the diagonal matrix

$$
D_{\mathcal{H}}:=\left(\begin{array}{cc}
D_{\mathcal{G}} & O_{l \times m} \\
O_{m \times l} & x^{\nu_{1}} D_{\mathcal{F}}
\end{array}\right)
$$

and we transform system (13) to (5) by he change $\mathbf{y}=D_{\mathcal{H}} \mathbf{Y}$, where $\mathbf{Y}$ is the unknown vector function with $m+l$ components, that is, $\mathbf{Y}$ satisfies the system of equations

$$
x \frac{d \mathbf{Y}}{d x}=\left(A_{\mathcal{H}}+B_{\mathcal{H}}(x)\right) \mathbf{Y} .
$$

Here the scalar matrix $A_{\mathcal{H}}$ and the matrix function $B_{\mathcal{H}}(x)$ are determined by the identities

$$
A_{\mathcal{H}}=\left(\begin{array}{cc}
\tilde{A}_{\mathcal{G}} & M \\
O_{m \times l} & \tilde{A}_{\mathcal{F}}
\end{array}\right), \quad B_{\mathcal{H}}(x)=\left(\begin{array}{cc}
\tilde{B}_{\mathcal{G}}(x) & O_{l \times m} \\
S(x) & \tilde{B}_{\mathcal{F}}(x)
\end{array}\right)
$$

where the matrices $\tilde{A}_{\mathcal{G}}, \tilde{A}_{\mathcal{F}}$ and the matrix functions $\tilde{B}_{\mathcal{G}}, \tilde{B}_{\mathcal{F}}$ are defined via the procedure applied in Section 2, see equation (5)), and $S(x)$ is a matrix of size $m \times l$ and all its elements vanish except the element $i^{-m-l} \lambda / x^{\nu_{1}+\nu_{2}}$ in the left lower corner.

It is clear that the detailed description of the matrices $D_{\mathcal{H}}, A_{\mathcal{H}}$ and $B_{\mathcal{H}}(x)$ depend on the parity of the numbers $l$ and $m$ and would take a lot of place. This is why we restrict ourselves by what has been said above.

Let $r+1$ be the maximal size of the Jordan block in the canonical form of the matrix $A_{\mathcal{H}}$ and let the number $r$ and the matrix $B(x)\left(=B_{\mathcal{H}}(x)\right)$ satisfy Condition $(\mathrm{C})$, see (6). Then, as in Section 3, we can apply Lemma 1 and prove the analogues of Theorem 1 and Corollary 1 for equation (3) in the case $\tau=\tau_{\mathcal{H}}$, that is, for the equation

$$
\tau_{\mathcal{F}} \tau_{\mathcal{G}} y=\lambda y .
$$

Remark 5. We can show that the characteristic polynomial $\mathfrak{F}_{\mathcal{H}}\left(z, \nu_{1}, \nu_{2}\right)$ of the matrix $A_{\mathcal{H}}$ is determined by the identity

$$
\mathfrak{F}_{\mathcal{H}}\left(z, \nu_{1}, \nu_{2}\right)=\mathfrak{F}_{\mathcal{G}}\left(z, \nu_{1}\right) \mathfrak{F}_{\mathcal{F}}\left(z+\nu_{1}, \nu_{2}\right),
$$

and the number $r+1$ is the maximal multiplicity of the roots of the product of these polynomials. In particular, in the case $\mathcal{G}=\mathcal{F}=F$ (for the definition of the matrix $F$ see Section 2) and, respectively, $\nu_{1}=\nu_{2}=\nu$, the characteristic polynomial for the square of the expression $\tau_{F}^{2}$ is defined by the identity

$$
\mathfrak{F}_{\mathcal{H}}(z, \nu)=\mathfrak{F}_{F}(z, \nu) \mathfrak{F}_{F}(z+\nu, \nu),
$$

where, according to Remark 4 , the polynomial $\mathfrak{F}_{F}(z, \nu)$ is such that

$$
\mathfrak{F}_{F}(z, \nu)=\mathfrak{F}_{2 n}(z, \nu) \quad \text { or } \quad \mathfrak{F}_{F}(z, \nu)=\mathfrak{F}_{2 n+1}(z, \nu) .
$$

We observe that here the conditions $\nu_{1}, \nu_{2}>0$ were needed for the sake of the brevity of the exposition and they can be replaced by the conditions $\nu_{1}, \nu_{2} \geqslant 0$. Moreover, the method proposed in this section for the product of two expression is generalized with no problems for the product of finitely many quasi-differential expressions. 


\section{DEFICIENCY INDEX FOR THE MINIMAL OPERATOR}

5.1. In this section we assume that apart of Conditions 1) and 2) (cf. Introduction), the matrix $F$ satisfies also the condition:

3) $F=-J^{-1} F^{*} J$, where $F^{*}$ is the adjoint matrix of $F$ and

$$
J:=\left((-1)^{i} \delta_{i, m+1-j}\right), \quad i, j=1,2, \ldots, m,
$$

$\delta_{i j}$ is the Kronecker delta.

This condition ensures the validity of the Lagrange formula for the quasi-differential expression $\tau y$ (see (2)), namely, for all functions $u, v \in \mathcal{D}(\tau)$, the identity

$$
\int_{\alpha}^{\beta} \bar{v} \tau u-\int_{\alpha}^{\beta} u \bar{\tau} \bar{v}=[u, v](\beta)-[u, v](\alpha), \quad \alpha, \beta \in(a, b),
$$

hold true, where

$$
[u, v](x)=i^{m} \sum_{j=0}^{m-1}(-1)^{m+1-j} u^{[j]}(x) \overline{v^{[m-1-j]}(x)},
$$

and the quasi-derivatives $y^{[j]}(j=0,1, \ldots, m-1)$ are defined by formulae (1).

Following the well-known procedure (see, for instance, [1, Sect. I]), we define the minimal closed symmetric operator $L_{0}$ generated by the expression $\tau y$ in the Hilbert space $\mathcal{L}^{2}[1,+\infty)$. By $D_{0}^{\prime}$ we denote the set of all complex-valued compactly supported functions on $[1,+\infty)$ in $\mathcal{D}(\tau)$ such that $\tau y \in \mathcal{L}^{2}[1,+\infty)$. It was established in work [1, Appen. A] that the set $D_{0}^{\prime}$ is everywhere dense in $\mathcal{L}^{2}[1,+\infty)$ and by the formula $L_{0}^{\prime} y=\tau y$, the expression $\tau y$ defines a symmetric (non-closed) operator in $\mathcal{L}^{2}[1,+\infty)$ on the domain $D_{0}^{\prime}$. By the symbols $L_{0}$ and $D_{0}$ we denote the closure of this operator and its domain, respectively.

Thus, in the considered case, the expression $\tau y$ generates the minimal closed symmetric operator $L_{0}$ in the Hilbert space $\mathcal{L}^{2}[1,+\infty)$. This is why the expression $\tau y$ is called symmetric (formally self-adjoint) quasi-differential expression generated by the matrix $F$.

Let $\lambda$ be a complex number with a non-zero imaginary part, $\operatorname{Im} \lambda \neq 0$. By $R_{\lambda}$ and $R_{\bar{\lambda}}$ we denote the range of the operators $L_{0}-\lambda I$ and $L_{0}-\bar{\lambda} I$, respectively, ( $I$ is the identity mapping), while by $\mathcal{N}_{\lambda}$ and $\mathcal{N}_{\bar{\lambda}}$ we denote their orthogonal complements in the space $\mathcal{L}^{2}[1,+\infty)$. The spaces $\mathcal{N}_{\lambda}$ and $\mathcal{N}_{\bar{\lambda}}$ are called defect subspaces associated with the numbers $\lambda$ and $\bar{\lambda}$. Their dimensions $\operatorname{dim} \mathcal{N}_{\lambda}$ and $\operatorname{dim} \mathcal{N}_{\bar{\lambda}}$ are same in the upper and lower half-planes. We denote $n_{+}=\operatorname{dim} \mathcal{N}_{\lambda}$ and $n_{-}=\operatorname{dim} \mathcal{N}_{\bar{\lambda}}$ as $\operatorname{Im} \lambda>0$. The pair $\left(n_{+}, n_{-}\right)$is called the deficiency index of the operator $L_{0}$. It is known that the numbers $n_{+}$and $n_{-}$coincide with the maximal number of linearly independent solutions to equation (3) belonging to the space $\mathcal{L}^{2}[1,+\infty)$ as the parameter $\lambda$ is taken in the upper $(\operatorname{Im} \lambda>0)$ or lower $(\operatorname{Im} \lambda<0)$ half-plane, respectively. As $m=2 n$, the numbers $n_{+}$and $n_{-}$satisfy the inequalities

$$
n \leqslant n_{+}, n_{-} \leqslant m
$$

while as $m=2 n+1$, they satisfy the inequalities

$$
n \leqslant n_{+} \leqslant m, \quad n+1 \leqslant n_{-} \leqslant m, \quad \text { or } \quad n+1 \leqslant n_{+} \leqslant m, \quad n \leqslant n_{-} \leqslant m .
$$

5.2. Assume now that the entries of the matrix $F\left(F_{2 n}\right.$ or $\left.F_{2 n+1}\right)$ obey Conditions $(\mathrm{A})-(\mathrm{C})$ and, in addition, Condition 3). The according to Section 3, Theorem 1 and Corollary 1 are true for equation (3) and, moreover, according to Subsection 5.1, the expression $\tau y$ generates the minimal closed symmetric operator $L_{0}$ in the Hilbert space $\mathcal{L}^{2}[1,+\infty)$. Thus, Corollary 1 is the statement on the deficiency index of the operator $L_{0}$, that is, the following theorem is true. 
Theorem 2. Let the entries of the matrix $F\left(F_{2 n}\right.$ or $\left.F_{2 n+1}\right)$ satisfy Conditions $(A)-(C)$ and Condition 3). Then as $\nu>0$, the deficiency numbers of the operator $L_{0}$ coincide and are equal to the numbers of the polynomial $\mathfrak{F}(z, \nu)$ (see Remark 4) lying in the domain $\operatorname{Re} z<0$.

We can formulate and prove a theorem similar to Theorem 2 for the case were the quasidifferential expression $\tau y$ is the product of two quasi-differential expressions (see Section 4 and, in particular, Remark 5).

\section{BIBLIOGRAPHY}

1. W.N. Everitt, L. Marcus. Boundary value problems and sumpletic algebra for ordinary differential and quasi-differential operators. Math. Surv. Monog. Amer. Math. Soc. Providence, RI. 61, (1999).

2. W.N. Everitt. Linear ordinary quasi-differential equations. Lecture notes for the Fourth International Symposium on Differential equations and Differential Geometry. Univ. of Peking, Beijing. 1-28 (1986).

3. K.A. Mirzoev, A.A. Shkalikov. Differential operators of even order with distribution coefficients // Matem. Zamet. 99:5, 788-793 (2016). [Math. Notes. 99:5, 779-784 (2016).]

4. W.N. Everitt, A. Zettl. Products of differential expressions without smoothness assumptions // Quaestiones Mathematicae. 3:1, 67-82 (1978).

5. K.A. Mirzoev, N.N. Konechnaja. Asymptotics of solutions of a class of linear differential equations with nonsmooth coefficients // Matem. Zamet. 100:2, 321-317 (2016). [Math. Notes. 100:2, 334340 (2016).]

6. K.A. Mirzoev. Orlov's theorem on the deficiency index of differential operators // Dokl. Akad. Nauk. 380:5, 591-595 (2001). [Dokl. Math. 64:2, 236-240 (2001).]

7. I.N. Dolgikh, K.A. Mirzoev. Deficiency indices and spectrum of self-adjoint extensions of some classes of differential operators // Matem. Sborn. 197:4, 53-74 (2006). [Sb. Math. 197:4, 525-546 (2006).]

8. O. Dunkel. Regular singular points of a system homogeneous linear differential equations of the first order // American Acad. Proc. 38, 341-370 (1902)

9. S. Faedo. Proprieta asintotiche delle soluzioni dei sistemi differenziali lineari omogenei // Ann. Mat. Pura Appl. Ser. IV. 26, 207-215 (1947).

10. E.A. Coddington, N. Levinson. Theory of ordinary differential equations. McGill-Hill Book Company, New York (1955).

11. W.A. Coppel. Stability and asymptotic behavior of differential equations. Heath Mathematical Monographs. D.C. Heath and Company, Boston (1965).

Konechnaja Natalia Nikolaevna,

Northern (Arctic) Federal University

named after M.V. Lomonosov,

Severnaya Dvina Emb. 17,

163002, Arkhangelsk, Russia

E-mail: n.konechnaya@narfu.ru

Karakhan Agahan ogly Mirzoev,

Lomonosov Moscow State University,

Leninskie Gory, 1,

119991, Moscow, Russia

E-mail: mirzoev.karahan@mail.ru 